\title{
Multigrid preconditioners for symmetric Sinc systems
}

\author{
Michael K. Ng* Stefano Serra-Capizzano ${ }^{\dagger}$ \\ Cristina Tablino-Possio
}

(Received 8 August 2003; revised 9 January 2004)

\begin{abstract}
The symmetric Sinc-Galerkin method applied to a separable secondorder self-adjoint elliptic boundary value problem gives rise to a system of linear equations $\left(\Psi_{x} \otimes D_{y}+D_{x} \otimes \Psi_{y}\right) \mathbf{u}=\mathbf{g}$ where $\otimes$ is the Kronecker product symbol, $\Psi_{x}$ and $\Psi_{y}$ are Toeplitz-plus-diagonal matrices, and $D_{x}$ and $D_{y}$ are diagonal matrices. The main contribution of this paper is to present a two-step preconditioning strategy based on the banded matrix approximation and the multigrid iteration for these Sinc-Galerkin systems. Numerical examples show that the multigrid
\end{abstract}

*Department of Mathematics, The University of Hong Kong, Pokfulam Road, Hong Kong. mailto:mng@maths.hku.hk

†Dip. di Chimica, Fisica e Matematica, Universita' dell'Insubria - sede di Como, via Valleggio, 11, 22100 Como, Italy.

${ }^{\ddagger}$ Dip. di Matematica e Applicazioni, Università di Milano Bicocca, Via Bicocca degli Arcimboldi 8, 20126 Milano, Italy.

See http://anziamj.austms.org.au/V45/CTAC2003/Ngxx/home.html for this article, (C) Austral. Mathematical Soc. 2004. Published August 14, 2004. ISSN 1446-8735 
preconditioner is practical and efficient to precondition the conjugate gradient method for solving the above symmetric Sinc-Galerkin linear system.

\section{Contents}

1 Introduction

C858

2 Symmetric Sinc-Galerkin linear systems

C860

3 Multigrid preconditioners

C862

4 Numerical results

C865

References

C868

\section{Introduction}

We present a two-step preconditioner which is a technical hybrid of the banded matrix approximation and the multigrid iteration, for solving systems of linear equations arising from symmetric Sinc-Galerkin discretization of a separable second order self-adjoint elliptic boundary value problem

$$
\begin{cases}\mathcal{L} u=-\nabla^{2} u(x, y)+(\mu(x)+\nu(y)) u(x, y)=f(x, y), & (x, y) \in \Omega, \\ u(x, y)=0, & (x, y) \in \partial \Omega,\end{cases}
$$

where $\Omega$ is a rectangular region in the plane $\mathbb{R}^{2}, \partial \Omega$ its boundary, and $\mu(x)$, $\nu(y)$ and $f(x, y)$ are given nonlinear functions. Without loss of generality, we assume that $\Omega$ is the unit square $(0,1) \times(0,1)$. According to Lund [7] and Lund and Bowers [8] the $d_{x} d_{y} \times d_{x} d_{y}$ matrix system is of the form

$$
\left(\Psi_{x} \otimes D_{y}+D_{x} \otimes \Psi_{y}\right) \mathbf{u}=\mathbf{g},
$$


where $P \otimes Q$ represents the Kronecker product of two matrices $P$ and $Q$, $\Psi_{x}$ and $\Psi_{y}$ are symmetric Toeplitz-plus-diagonal matrices, $D_{x}$ and $D_{y}$ are diagonal matrices, and $d_{x}$ and $d_{y}$ are the numbers of the mesh-points along the $x$ - and the $y$-directions, respectively. The attraction of this approach is that the symmetric Sinc-Galerkin method for boundary value problems is convergent exponentially under suitable conditions, and it is also effective in handling singular problems [7, 11].

Lund [7] used block Gauss elimination to solve the system of linear equations (2) through finding an orthogonal matrix which transforms the coefficient matrix into a block-diagonal one, and then solving the reduced system of linear equations by a block Gauss elimination process. This approach performs well because all matrix inversions and matrix multiplications in the Gauss elimination process can be performed only on diagonal matrices. Its cost for solving the symmetric Sinc-Galerkin linear system (2) is of $\mathcal{O}\left(d_{x} d_{y}^{3}\right)$ or $\mathcal{O}\left(d_{x}^{3} d_{y}\right)$ operations.

Recently, Ng [9] constructed a banded-matrix preconditioner

$$
B=B_{x} \otimes D_{y}+D_{x} \otimes B_{y}
$$

to precondition the coefficient matrix in (2) and employed the resulting preconditioned conjugate gradient method to solve the linear system (2). Here $B_{x}$ and $B_{y}$ are tridiagonal matrices. All eigenvalues of the preconditioned matrix are uniformly bounded by positive constants from below and above, respectively [9], and therefore, the condition number of the preconditioned matrix is uniformly bounded by a constant independent of the matrix size. Considering that the computational complexity of solving $B \mathbf{v}=\mathbf{r}$ is still quite expensive, $\mathrm{Ng}$ [9] further adopted the MINV [3] as a second-step preconditioner to precondition the matrix $B$ in numerical experiments. Numerical results showed that the preconditioned conjugate gradient method with the two-step preconditioner composed of the banded matrix $B$ and its MINV factorization converges quite rapidly.

In this paper, we investigate the solution of the system of linear equa- 
tions (2) by the preconditioned conjugate gradient method with a two-step preconditioner which is a technical hybrid of the banded matrix $B$ and its multigrid iterations. Numerical examples show that the new preconditioner gives a practical and efficient to precondition the conjugate gradient method for solving the symmetric Sinc-Galerkin linear system (2). In Section 2 we study some properties of the discrete Sinc-Galerkin linear system. In Section 3 we introduce the new two-step preconditioner. Numerical results are presented in Section 4 to illustrate the effectiveness of the new preconditioner.

\section{Symmetric Sinc-Galerkin linear systems}

We solve the system of linear equations (2) by the preconditioned conjugate gradient method, in which the matrix-vector product is the most costly part in each iteration. However, for the special structure of the coefficient matrix in the system of linear equations (2) we show that the matrix-vector product can be computed efficiently.

By assembling the Sinc-Galerkin linear system for the coefficients, we obtain the system of linear equations

$$
A \mathbf{u}=\left[\left(T_{x}+\Phi_{x}\right) \otimes D_{y}+D_{x} \otimes\left(T_{y}+\Phi_{y}\right)\right] \mathbf{u}=\mathbf{g},
$$

where

$$
\begin{gathered}
\mathbf{u}=\left[u_{1,1}, u_{1,2}, \ldots, u_{1, m_{y}+n_{y}+1}, \ldots, u_{m_{x}+n_{x}+1, m_{y}+n_{y}+1}\right]^{T} ; \\
\mathbf{g}=\left[g_{1,1}, g_{1,2}, \ldots, g_{1, m_{y}+n_{y}+1}, \ldots, g_{m_{x}+n_{x}+1, m_{y}+n_{y}+1}\right]^{T} ; \\
\Phi_{x}=h_{x}^{2} \cdot \operatorname{diag}\left[\left(\frac{-1}{\phi^{\prime}\left(x_{-m_{x}}\right)^{3 / 2}}\right)\left(\frac{1}{\phi^{\prime}\left(x_{-m_{x}}\right)^{1 / 2}}\right)^{\prime \prime}+\frac{\mu\left(x_{-m_{x}}\right)}{\phi^{\prime}\left(x_{-m_{x}}\right)^{2}}, \ldots,\right. \\
\left.\left(\frac{-1}{\phi^{\prime}\left(x_{n_{x}}\right)^{3 / 2}}\right)\left(\frac{1}{\phi^{\prime}\left(x_{n_{x}}\right)^{1 / 2}}\right)^{\prime \prime}+\frac{\mu\left(x_{n_{x}}\right)}{\phi^{\prime}\left(x_{n_{x}}\right)^{2}}\right]
\end{gathered}
$$




$$
\begin{gathered}
\Phi_{y}=h_{y}^{2} \cdot \operatorname{diag}\left[\left(\frac{-1}{\phi^{\prime}\left(y_{-m_{y}}\right)^{3 / 2}}\right)\left(\frac{1}{\phi^{\prime}\left(y_{-m_{y}}\right)^{1 / 2}}\right)^{\prime \prime}+\frac{\nu\left(y_{-m_{y}}\right)}{\phi^{\prime}\left(y_{-m_{y}}\right)^{2}}, \ldots,\right. \\
\left.\left(\frac{-1}{\phi^{\prime}\left(y_{n_{y}}\right)^{3 / 2}}\right)\left(\frac{1}{\phi^{\prime}\left(y_{n_{y}}\right)^{1 / 2}}\right)^{\prime \prime}+\frac{\nu\left(y_{n_{y}}\right)}{\phi^{\prime}\left(y_{n_{y}}\right)^{2}}\right]
\end{gathered}
$$

with $\phi(\cdot)$ a conformal mapping $[7,8]$,

$$
x_{j}=\phi^{-1}\left(j h_{x}\right), \quad y_{k}=\phi^{-1}\left(k h_{y}\right), \quad j, k=0, \pm 1, \pm 2, \ldots ;
$$

and for $z \in\{x, y\}, d_{z}=m_{z}+n_{z}+1, T_{z}$ is a $d_{z} \times d_{z}$ symmetric Toeplitz matrix with its first column

$$
\left[\pi^{2} / 3,-2,2 / 2^{2}, \ldots, 2(-1)^{d_{z}-1} /\left(d_{z}-1\right)^{2}\right]^{T}
$$

and

$$
D_{z}=\operatorname{diag}\left[\frac{1}{\phi^{\prime}\left(z_{-m_{z}}\right)^{1 / 2}}, \ldots, \frac{1}{\phi^{\prime}\left(z_{n_{z}}\right)^{1 / 2}}\right]
$$

Evidently, the coefficient matrix $A$ in (4) is a block Toeplitz-plus-diagonal matrix. By sufficiently considering this special structure, we see that the matrix-vector product $A \mathbf{w}$ should be computed in $\mathcal{O}\left(d_{x} d_{y} \log \left(d_{x} d_{y}\right)\right)$ operations [9]. Thus, iterative methods such as the conjugate gradient method may be efficient in solving this system of linear equations. In general, the convergence rate of the conjugate gradient method depends on the condition number of the coefficient matrix $A$. Also, $\mathrm{Ng}$ [9] showed that the condition number of the matrix $A$ is of order $\mathcal{O}\left(d_{x}^{2}+d_{y}^{2}\right)$, which implies that the conjugate gradient method may converge very slowly when it is employed to solve the system of linear equations (4).

To accelerate the convergence speed of the conjugate gradient method, $\mathrm{Ng}[9]$ constructed a banded matrix preconditioner

$$
B=\left(L_{x}+\Phi_{x}\right) \otimes D_{y}+D_{x} \otimes\left(L_{y}+\Phi_{y}\right)
$$


to the matrix $A$, and showed that this preconditioner is optimal in the sense of the spectral equivalence. Here $L_{x}$ (or $L_{y}$ ) is an one-dimensional discrete Laplacian matrix of the form

$$
L_{x}=\operatorname{tridiag}[-1,2,-1] .
$$

More precisely, we state the main result of $\mathrm{Ng}$ [9].

Theorem 1 If $\mu$ and $\nu$ in the separable second-order self-adjoint elliptic boundary value problem (1) are nonnegative, and the conformal mapping $\phi$ satisfies

$$
\left(\frac{-1}{\left(\phi^{\prime}(x)\right)^{3 / 2}}\right)\left(\frac{1}{\left(\phi^{\prime}(x)\right)^{1 / 2}}\right)^{\prime \prime} \geq 0, \quad \text { for all } x \in(0,1),
$$

then $L_{x}+\Phi_{x}, L_{y}+\Phi_{y}, D_{x}, D_{y}$ and $B$ are symmetric positive definite matrices. In addition, all eigenvalues of the matrix $B^{-1} A$ are within an interval located on the positive semi-axis, and therefore, the condition number $\kappa\left(B^{-1} A\right)$ of the matrix $B^{-1} A$ is uniformly bounded by a positive constant $c$ independent of the matrix size.

In fact, the matrix $B$ is a block-tridiagonal matrix. However, the work for solving the generalized residual system

$$
B \mathbf{v}=\mathbf{r}
$$

for some vector $\mathbf{r}$, is quite costly. In the next section, we propose a multigrid strategy for solving (9).

\section{$3 \quad$ Multigrid preconditioners}

Consider two basic iterations

$$
\mathbf{x}^{(j+1)}=V^{(i)} \mathbf{x}^{(j)}+\mathbf{b}^{(i)}:=\mathcal{V}^{(i)}\left(\mathbf{x}^{(j)}, \mathbf{b}^{(i)}\right), \quad i=1,2,
$$


for the solution of the linear system $A \mathbf{x}=\mathbf{b}$, where $A, M^{(i)}, V^{(i)}:=I-$ $\left[M^{(i)}\right]^{-1} A \in \mathbf{C}^{n \times n}$, and $\mathbf{b}, \mathbf{b}^{(i)}:=\left[M^{(i)}\right]^{-1} \mathbf{b} \in \mathbf{C}^{n}$. Given a full-rank matrix $P^{k} \in \mathbf{C}^{n \times k}$, with $k<n$, a Two-Grid Method (TGM) is defined by the following algorithm [6]

$\operatorname{TGM}\left(V^{(1)}, V^{(2)}, P^{k}, \nu_{1}, \nu_{2}\right)\left(\mathbf{x}^{(j)}\right)$

1. $\tilde{\mathbf{x}}^{(j)}=\left[\mathcal{V}^{(1)}\right]^{\nu_{1}}\left(\mathbf{x}^{(j)}, \mathbf{b}^{(1)}\right)$

2. $\tilde{\mathbf{d}}=A \tilde{\mathbf{x}}^{(j)}-\mathbf{b}$

3. $\mathbf{d}=\left(P^{k}\right)^{*} \tilde{\mathbf{d}}$

4. $A_{k}=\left(P^{k}\right)^{*} A P^{k}$

5. Solve $A_{k} \mathbf{y}=\mathbf{d}$

6. $\hat{\mathbf{x}}^{(j)}=\tilde{\mathbf{x}}^{(j)}-P^{k} \mathbf{y}$

7. $\mathbf{x}^{(j+1)}=\left[\mathcal{V}^{(2)}\right]^{\nu_{2}}\left(\hat{\mathbf{x}}^{(j)}, \mathbf{b}^{(2)}\right)$

In the algorithm, $*$ denotes the Hermitian transpose. Steps 1 and 7 concern the application of $\nu_{1}$ sweeps of the "intermediate iteration" (10) with $i=1$ and of $\nu_{2}$ steps of the "smoothing iteration" (10) with $i=2$, whereas steps 2-6 define the "coarse grid correction" that depends on the projection operator $P^{k}$. Here the names "intermediate" and "smoothing" refer to the multi-iterative terminology [10]. We say that a method is multiiterative if it is composed of at least two distinct iterations: the idea is that these basic components should have complementary spectral behaviours so that the whole procedure is fast. In our case the "smoothing iteration" is chosen in such a way that it reduces the error in the subspace where $A$ is well-conditioned; (but it is slowly convergent in the rest of the space) the coarse grid iteration has spectral radius equal to 1 and therefore does not 
converge at all, but it is very rapidly convergent in the subspace where $A$ is ill-conditioned; finally the "intermediate" iteration is strongly convergent in that subspace where the combined effect of the other two iterations resulted to be less effective.

The global iteration matrix of $\mathrm{TGM}:=\mathrm{TGM}^{k}$ is then

$$
\operatorname{TGM}\left(V, P^{k}\right)=\left[V^{(2)}\right]^{\nu_{2}}\left[I-P^{k}\left(\left(P^{k}\right)^{*} A P^{k}\right)^{-1}\left(P^{k}\right)^{*} A\right]\left[V^{(1)}\right]^{\nu_{1}} .
$$

Fiorentino and Serra-Capizzano $[4,5]$ proposed a fast TGM and a fast multigrid (V-cycle) method for Toeplitz and $\tau$ problems (the $\tau$ class is the algebra associated to the most known sine transform [1]). In this paper, we consider the smoothing iteration (10), where the matrix

$$
V^{(1)}=I-B /\|B\|_{\infty},
$$

and the intermediate iteration is

$$
V^{(2)}=I-2 B /\|B\|_{\infty} .
$$

Following $[4,5,2]$, the projection is constructed as

$$
P_{k}=(\operatorname{tridiag}[1,2,1] \otimes \operatorname{tridiag}[1,2,1]) U^{k},
$$

where the matrix

$$
U^{k}=T_{n_{1}}^{k_{1}} \otimes T_{n_{2}}^{k_{2}},
$$

with $n_{j}=2 k_{j}+1$ and the matrix

$$
\left(T_{n}^{k}\right)_{i, j}= \begin{cases}1, & \text { for } i=2 j-1, j=1, \ldots, k \\ 0, & \text { otherwise }\end{cases}
$$

Numerical results in the next section show that the proposed multigrid preconditioners are efficient. 


\section{Numerical results}

The two examples from $\mathrm{Ng}$ [9] illustrate the performance of the new hybrid multigrid preconditioner.

\section{Example 2}

$-\nabla^{2} u(x, y)=3 e^{x+y} x y[(x+3)(1-y)+(y+3)(1-x)], \quad(x, y) \in(0,1) \times(0,1)$.

The known solution is $u(x, y)=3 e^{x+y} x y(1-x)(1-y)$. The conformal mapping $\phi(z)=\log (z /(1-z))$ is used in this example.

\section{Example 3}

$$
\begin{gathered}
-\nabla^{2} u(x, y)+\left(\frac{1}{x^{2}}+\frac{1}{y^{2}}\right) u(x, y)=\frac{y \ln (y)(\ln (x)-1)}{x}+\frac{x \ln (x)(\ln (y)-1)}{y}, \\
(x, y) \in(0,1) \times(0,1) .
\end{gathered}
$$

The solution is $u(x, y)=x y \ln (x) \ln (y)$. Here, the solution has logarithmic singularities at $x=0$ or $y=0$. The conformal mapping $\phi(z)=\log (z /(1-z))$ is also used in this example.

In these two examples, $\phi$ satisfies the conditions of Theorem 1. In the test, we take $d=\pi / 2$ and $m_{x}=n_{x}=m_{y}=n_{y}=m$ as suggested in Lund [7], where the size of the Sinc-Galerkin matrix is $(2 m+1)^{2}$. Moreover, the problems all have homogeneous Dirichlet boundary conditions and known solutions.

All the experiments are performed in MATLAB with machine precision $10^{-16}$. In our tests, the zero vector is the initial guess of the preconditioned conjugate gradient iterations. We note that the accuracy of the computed solution depends on the Sinc-Galerkin method used in the discretization of 
TABLE 1: Number of iterations required for convergence for Example 2 when $\alpha=\beta=1 / 2$ and $h=\pi / \sqrt{m}$.

\begin{tabular}{ccccc}
\hline Size & Error & I & B & M \\
\hline $9^{2}$ & $4.1 \times 10^{-3}$ & 19 & 3 & 3 \\
$17^{2}$ & $3.7 \times 10^{-4}$ & 46 & 3 & 3 \\
$33^{2}$ & $1.1 \times 10^{-5}$ & $* *$ & 4 & 4 \\
$65^{2}$ & $7.6 \times 10^{-8}$ & $* *$ & 5 & 5 \\
\hline
\end{tabular}

TABLE 2: Number of iterations required for convergence for Example 2 when $\alpha=\beta=1$ and $h=\pi / \sqrt{2 m}$.

\begin{tabular}{ccccc}
\hline Size & Error & I & B & M \\
\hline $9^{2}$ & $1.5 \times 10^{-2}$ & 22 & 3 & 3 \\
$17^{2}$ & $3.3 \times 10^{-3}$ & 46 & 4 & 4 \\
$33^{2}$ & $3.0 \times 10^{-4}$ & $>100$ & 5 & 5 \\
$65^{2}$ & $8.9 \times 10^{-6}$ & $>100$ & 6 & 6 \\
\hline
\end{tabular}

the boundary value problem. We determine the error between the numerical approximation and the true solution at the Sinc points defined as follows:

$$
\text { Error }=\max _{-m_{x} \leq j \leq n_{x},-m_{y} \leq k \leq n_{y}}\left|u_{j, k}-u\left(x_{j}, y_{k}\right)\right| .
$$

These error numbers were computed by $\mathrm{Ng}$ [9] by a direct solver applied to the relevant Sinc-Galerkin system. One cannot expect iterative methods to solve the underlying problem more accurately, so we stop the iterations when the error between numerical iterate and true solution at the Sinc nodes is comparable to the error computed by the direct solver.

Tables 1-4 list the number of iterations required for the convergence of the conjugate gradient method using the hybrid preconditioner. Two sets of parameters: (a) $\alpha=\beta=1 / 2$ and $h=\pi / \sqrt{m}$, and (b) $\alpha=\beta=1$ and $h=\pi / \sqrt{2 m}$, are used to test the method (see Lund [7]). In the tables, the symbol I means that the system is solved without using a preconditioner, 
TABLE 3: Number of iterations required for convergence for Example 3 when $\alpha=\beta=1 / 2$ and $h=\pi / \sqrt{m}$.

\begin{tabular}{ccccc}
\hline Size & Error & I & B & M \\
\hline $9^{2}$ & $6.6 \times 10^{-4}$ & 19 & 4 & 4 \\
$17^{2}$ & $1.1 \times 10^{-4}$ & 46 & 5 & 5 \\
$33^{2}$ & $5.8 \times 10^{-6}$ & $>100$ & 8 & 6 \\
$65^{2}$ & $6.0 \times 10^{-8}$ & $>100$ & 13 & 9 \\
\hline
\end{tabular}

TABLE 4: Number of iterations required for convergence for Example 3 when $\alpha=\beta=1$ and $h=\pi / \sqrt{2 m}$.

\begin{tabular}{ccccc}
\hline Size & Error & I & B & M \\
\hline $9^{2}$ & $5.1 \times 10^{-3}$ & 19 & 6 & 5 \\
$17^{2}$ & $1.7 \times 10^{-3}$ & 46 & 8 & 5 \\
$33^{2}$ & $2.2 \times 10^{-4}$ & $>100$ & 15 & 6 \\
$65^{2}$ & $9.6 \times 10^{-6}$ & $>100$ & 24 & 9 \\
\hline
\end{tabular}

the symbol $\mathbf{B}$ represents the banded MINV preconditioner that was used by $\mathrm{Ng}$ [9], and the symbol $\mathbf{M}$ represents that the system is solved using the new hybrid preconditioner. The used multigrid algorithm is the $\mathrm{V}$-cycle with direct solution of the system when the size equals $9^{2}$.

See that as the size increases, the number of conjugate gradient iterations increases if no preconditioner is used. In Example 3, when the size is $33^{2}$ or $65^{2}$, the numbers of conjugate gradient iterations required for convergence exceed 100 iterations. However, all the preconditioned systems converge in less than 100 iterations. For Example 3, we find that the performance of hybrid preconditioners is better since our preconditioning strategy is based on the banded matrix approximation and the banded matrix approximation is better than MINV preconditioning for variable coefficient problems.

Finally, we conclude that the main contribution of this paper is to present a two-step preconditioning strategy based on the banded matrix approxima- 
tion and the multigrid iteration for these symmetric Sinc-Galerkin linear systems. Our numerical results show that the two-step preconditioner is practical and efficient to precondition the conjugate gradient method for solving the symmetric Sinc-Galerkin linear systems.

Acknowledgments: Research supported in part by RGC Grant Nos. 7130/ 02P and 7046/03P, and HKU CRCG Grant Nos. 10203501, 10203907 and 10203408 .

\section{References}

[1] D. Bini and M. Capovani, Spectral and computational properties of band symmetric Toeplitz matrices, Linear Algebra Appl., 52/53 (1983), pp. 99-125. C864

[2] R. Chan, Q. Chang and H. Sun, Multigrid Method for Ill-conditioned Symmetric Toeplitz Systems, SIAM J. Sci. Comput., 19 (1998), pp. 516-529. C864

[3] P. Concus, G. Golub, and G. Meurant, Block Preconditioning for the Conjugate Gradient Method, SIAM J. Sci. Statist. Comput., 6 (1985), pp. 220-252. C859

[4] G. Fiorentino and S. Serra-Capizzano, Multigrid Methods for Toeplitz Matrices, Calcolo, 28 (1991), pp. 283-305. C864

[5] G. Fiorentino and S. Serra-Capizzano, Multigrid Methods for Symmetric Positive Definite Block Toeplitz Matrices with Nonnegative Generating Functions, SIAM J. Sci. Comput., 17 (1996), pp. 1068-1081. C864 
[6] W. Hackbusch, Multigrid Methods and Applications, Springer Verlag, Berlin, 1985. C863

[7] J. Lund, Symmetrization of the Sinc-Galerkin Method for Boundary Value Problems, Math. Comput., 47 (1986), pp. 571-588. C858, C859, C861, C865, C866

[8] J. Lund and K. Bowers, Sinc Methods for Quadrature and Differential Equations, SIAM, 1992. C858, C861

[9] M. Ng, Fast Iterative Methods for Symmetric Sinc-Galerkin Systems, IMA J. Numer. Anal., 19 (1999), pp. 357-373. C859, C861, C862, C865, C866, C867

[10] S. Serra-Capizzano, Multi-iterative methods, Comput. Math. Appl., 26 (1993), pp. 65-87. C863

[11] F. Stenger, Numerical Methods Based on Sinc and Analytic Functions, Springer Series in Comp. Math., Springer Verlag, 1993. C859 